Pacific Journal of Mathematics

NOTE ON $\boldsymbol{H}$-EQUIVALENCES 


\title{
A NOTE ON $H$-EQUIVALENCES
}

\author{
DONALD W. KaHN
}

If $X$ is a space, with base point, the set of homotopy classes of based self-equivalent maps, from $X$ to itself, forms a group, which has been studied by many authors. In this note, we study a related group, in the case where $X$ is an $H$-space. The main result is that all such groups are finitelypresented. The methods combine results from algebraic topology with combinatorial group theory.

If $X$ is an $H$-space with multiplication $\mu: X \times X \rightarrow X$, a self-map $f: X \rightarrow X$ is called an $H$-map if

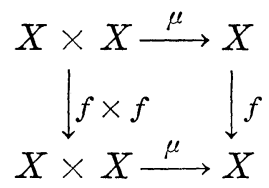

is homotopy commutative. Such maps were first studied in [6], and later in [1]. Arkowitz and Curjel [1] showed that if $X$ is a connected complex, which is an $H$-space, $X$ has finite-dimensional, commutative, rational Pontrjagin algebra, and the total homotopy groups of $X$ are finitely-generated, then the group of homotopy classes of self-maps, which are $H$-maps, is finitely-generated. We denote this group by $A(X)$, and remark that it is known to be frequently a complicated, non-Abelian group. Observe that this theorem of [1] suffices to handle the case when $X$ is a finite, connected complex, which is an $H$-space. The purpose of this note is to show how this result can be strengthened. We shall prove

THEOREM. If $X$ satisfies the assumptions of the theorem of Arkowitz and Curjel, then $A(X)$ is finitely-presented (see [3] for a definition).

The class of finitely-presented groups is countable, while it is known that there are uncountably many groups with 2 generators. (This result about uncountability, due to B. H. Neumann, may be found in [3]). Hence, our theorem narrows down the possibilities for $A(X)$ appreciably.

To prove this Theorem, we need several propositions.

Proposition 1. Let $N \subset G$ be a normal subgroup of the group $G$. 
Set $K=G / N$. If $K$ and $N$ are finitely presented, so is $G$.

Proof. See p. 130 in [2]. I believe that this is the first place where this proposition, which is not difficult, has appeared in the literature.

REMARK. On the contrary, if $G$ and $K$ are finitely-presented, $N$ need not even be finitely-generated.

Proposition 2. Let $H \subset G$ be a subgroup of finite index. If $G$ is finitely-presented, so is $H$.

Proof. See p. 93 of [4].

As a converse of Proposition 2, we have the following proposition which we shall deduce briefly from Proposition 1.

Proposition 3. If $H \subset G$ is a finitely-presented subgroup of finite index, then $G$ is finitely-presented.

Proof. Let $H_{0}$ be the intersection of all conjugates of $H$ in $G$. $H_{0}$ is a normal subgroup of finite-index, as there are only finitelymany conjugates. By Proposition 2, $H_{0}$ is finitely-presented. $G / H_{0}$ is finite, and hence, finitely-presented. The result follows immediately from Proposition 1.

Proposition 4. If $G_{1}, \cdots, G_{k}$ are finitely-presented, so is the group $\prod_{i=1}^{k} G_{i}$.

Proof. For lack of a reference, we indicate the proof. As generators, we select the elements

$$
\begin{aligned}
& \left(x_{1}, 1, \cdots, 1\right),\left(x_{2}, 1, \cdots, 1\right), \cdots, \\
& \left(1, y_{1}, 1, \cdots, 1\right), \cdots \quad,\left(1, x_{l}, 1, \cdots, 1\right) \\
& \quad, \cdots 1)
\end{aligned}
$$

where the $x_{i}$ generate $G_{1}$, the $y_{j}$ generate $G_{2}$, etc. A defining set of relations is then given by the relations among the $x_{i}$, the relations among the $y_{j}$, etc. plus the commutativity relations

$$
\left(x_{i}, 1, \cdots, 1\right) \cdot\left(1, y_{j}, 1, \cdots, 1\right)=\left(1, y_{j}, 1, \cdots, 1\right) \cdot\left(x_{i}, 1, \cdots, 1\right) \text { etc. }
$$

We now prove our Theorem.

(a) Let $k$ be the maximal dimension for which $H_{i}(X, Q) \neq 0$. Let $F \subset \pi_{*}^{\prime}(X)=\sum_{i=1}^{k} \oplus \pi_{i}(X)$ be the (graded) free subgroup. We shall denote, by $\operatorname{Aut}_{1}(G)$, the group of graded automorphism of the 
graded group $G$, reserving the symbol Aut for the usual group of automorphisms. According to [5.], if $F_{0}$ is a finitely-generated, free, Abelian group, Aut $\left(F_{0}\right)$ is finitely-presented. It is clear that $\operatorname{Aut}_{1}(F)$ is a direct product of such groups, and hence by Proposition 4, it is finitely-presented. Because $\operatorname{Aut}_{1}(F) \subset \operatorname{Aut}_{1}\left(\pi_{*}^{\prime}(X)\right)$ is clearly a subgroup of finite index, we conclude from Proposition 3 that the group $\operatorname{Aut}_{1}\left(\pi_{*}^{\prime}(X)\right)$ is finitely-presented.

(b) It is shown in [1] that the natural map

$$
P: A(X) \rightarrow \operatorname{Aut}_{1}\left(\pi_{*}^{\prime}(x)\right)
$$

has finite kernel, and that the image of $p$ (see p. 146 of [1]) is a subgroup of finite index. It is here that the assumptions on $X$ are used.

By (a) above, and Proposition 2, we see that $\operatorname{Im}(p)$ is finitelypresented. $\operatorname{ker}(p)$ being trivially finitely-presented, our theorem follows immediately from Proposition 1.

In conclusion, we would like to make some remarks about the full group of homotopy equivalences, $G(x)$, for such a space $X$. Clearly, we have a similar homomorphism $p_{1}$ and $\operatorname{Im}\left(p_{1}\right)$ is of finite-index. However, ker $p_{1}$ is no longer finite. For consider the space

$$
X=K(Z, 2 n) \times K(Z, 4 n) \quad n>0
$$

with the usual $H$-space structure. A self-map is determined up to homotopy by 2-cohomology classes, the classes $f^{*}\left(i_{2 n}\right)$ and $f^{*}\left(i_{4 n}\right)$, these being the images of the fundamental classes. We set, for any integer $k$,

$$
\begin{aligned}
& f_{k}^{*}\left(i_{2 n}\right)=i_{2 n} . \\
& f_{k}^{*}\left(i_{4 n}\right)=i_{4 n}+k\left(i_{2 n} \cup i_{2 n}\right) .
\end{aligned}
$$

It is easy to check that such a map $f_{k}$ induces the identity automorphism on homotopy groups, but that all the different $f_{k}$ represent distinct homotopy classes. Hence, the kernel of $p_{1}$ is infinite. An easy cohomology calculation shows that when $k \neq 0, f_{k}$ is not an $H$ map. One also see quickly that $A(X)$ does not have finite index in $G(X)$ in this case.

Nevertheless, one can prove that $G(X)$ is finitely-presented, by considering the kernel of $p_{1}$. This will be studied in the forthcoming thesis of Mr. Daniel Sunday.

\section{REFERENCES}

1. M. Arkowitz and C. Curjel, On maps of H-spaces Topology 6 No. 2 (1967).

2. H. Behr, Uber die endliche Definierbarkeit J. Reine u. Angew. Mathematik, 211 (1962). 
3. A. Kurosh, Theory of Groups Vol. 2, Chelsea Co., New York.

4. Magnus, Karass, and Solitar, Combinatorial Group Theory, J. Wiley, New York.

5. W. Magnus, Uber n-dim Gittertansformationen, Acta Math., 64 (1934).

6. H. Samelson, Groups and spaces of loops, Comm. Math. Helv., 28 (1954).

Received February 5, 1971 and in revised form June 24, 1971. Partially supported by NSF contract GP-8691.

The University of Minnesota 


\section{PACIFIC JOURNAL OF MATHEMATICS}

\section{EDITORS}

H. SAMELSON

Stanford University

Stanford, California 94305

C. R. HobBY

University of Washington

Seattle, Washington 98105
J. DUGUNDJI

Department of Mathematics

University of Southern California

Los Angeles, California 90007

RICHARD ARENS

University of California

Los Angeles, California 90024

\section{ASSOCIATE EDITORS}

E. F. BECKENBACH

B. H. NeumanN

F WoLF

K. YoshidA

\section{SUPPORTING INSTITUTIONS}

UNIVERSITY OF BRITISH COLUMBIA

UNIVERSITY OF SOUTHERN CALIFORNIA

CALIFORNIA INSTITUTE OF TECHNOLOGY

UNIVERSITY OF CALIFORNIA

MONTANA STATE UNIVERSITY

STANFORD UNIVERSITY

UNIVERSITY OF NEVADA

NEW MEXICO STATE UNIVERSITY

OREGON STATE UNIVERSITY

UNIVERSITY OF OREGON

OSAKA UNIVERSITY

UNIVERSITY OF TOKYO

UNIVERSITY OF UTAH

WASHINGTON STATE UNIVERSITY

UNIVERSITY OF WASHINGTON

$\stackrel{*}{*} \stackrel{*}{*} \stackrel{*}{*}{ }^{*}{ }^{*}$ MMEICAN MATHEMATICAL SOCIETY

NAVAL WEAPONS CENTER

Printed in Japan by International Academic Printing Co., Ltd., Tokyo, Japan 


\section{Pacific Journal of Mathematics}

\section{Vol. 42, No. $1 \quad$ January, 1972}

Tage Bai Andersen, On Banach space valued extensions from split faces ........

David Marion Arnold, A duality for quotient divisible abelian groups of finite

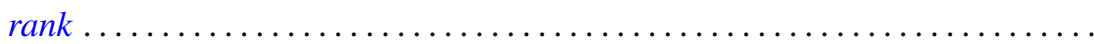

Donald Pollard Ballou, Shock sets for first order nonlinear hyperbolic

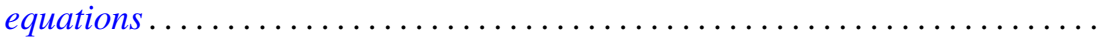

Leon Brown and Lowell J. Hansen, On the range sets of $H^{p}$ functions .........

Alexander Munro Davie and Arne Stray, Interpolation sets for analytic

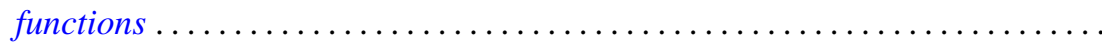

M. G. Deshpande, Structure of right subdirectly irreducible rings. II . . . . . . . . .

Barry J. Gardner, Some closure properties for torsion classes of abelian

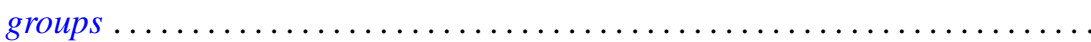

Paul Daniel Hill, Primary groups whose subgroups of smaller cardinality are

direct sums of cyclic groups . . . . . . . . . . . . . . . . . . .

Richard Allan Holzsager, When certain natural maps are equivalences .........

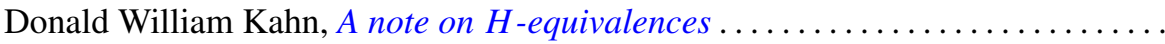

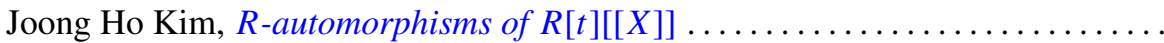

Shin'ichi Kinoshita, On elementary ideals of polyhedra in the 3-sphere.........

Andrew T. Kitchen, Watts cohomology and separability...

Vadim Komkov, A technique for the detection of oscillation of second order

ordinary differential equations .

Charles Philip Lanski and Susan Montgomery, Lie structure of prime rings of characteristic 2

Andrew Lenard, Some remarks on large Toeplitz determinants . .

Kathleen B. Levitz, A characterization of general Z.P.I.-rings. II .

Donald A. Lutz, On the reduction of rank of linear differential systems

David G. Mead, Determinantal ideals, identities, and the Wronskian ...

Arunava Mukherjea, A remark on Tonelli's theorem on integration in product

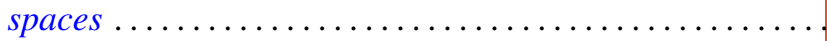

Hyo Chul Myung, A generalization of the prime radical in nonassociative rings.

John Piepenbrink, Rellich densities and an application to unconditionally nonoscillatory elliptic equations.

Michael J. Powers, Lefschetz fixed point theorems for a new class of multi-valued maps .

Aribindi Satyanarayan Rao, On the absolute matrix summability of a Fourier

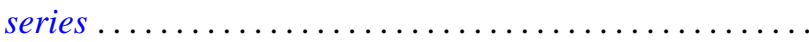

T. S. Ravisankar, On Malcev algebras ......................... 227

William Henry Ruckle, Topologies on sequences spaces . . . . . . . . . . . . . 235

Robert C. Shock, Polynomial rings over finite dimensional rings . . . . . . . . . 251

Richard Tangeman, Strong heredity in radical classes . . . . . . . . . . . . . . 259

B. R. Wenner, Finite-dimensional properties of infinite-dimensional spaces . . . . 267 